\title{
On One-Dimensional Integrable Quantum Systems with Infinitely Many Degrees of Freedom
}

\author{
S. N. M. RUIJSENAARs*† \\ Department of Physics, Princeton University, Princeton, New Jersey 08544
}

Received November 30, 1979

\begin{abstract}
We study one-dimensional quantum systems whose $S$-operator conserves the incoming momenta and particle identities. A survey of systems with known $S$-operators and an approach for solving them rigorously are presented, and several problems and conjectures are formulated. Subsequently, this approach is used to arrive at relativistic dynamics whose $S$-operators are those of the Ising model in the scaling limit and of the Federbush model. An invariance property of the wave and scattering operators is discovered and argued to hold at the classical level, too.
\end{abstract}

Contents. 1. Introduction. 2. Integrable systems: An overview and unified approach, 2A. Definition of integrability, 2B. Integrable systems with known $S$-matrices, $2 \mathrm{C}$. The Bethe Ansatz models, 2D. The positive energy relativistic field theories, 2E. A Bethe transform strategy, 2F. Comparison with the inverse scattering transform, 2G. A list of problems and conjectures. 3. An integrable $N$-particle system associated with pure transmission boundary conditions, 3A. Construction of the Bethe transforms, 3B. The results, 3C. The proofs. 4. Relativistic dynamics leading to the Ising and Federbush S-matrices, 4A. The continuum Ising model, 4B. The Federbush model.

\section{INTRODUCTION}

In this paper we study one-dimensional completely integrable quantum systems with infinitely many degrees of freedom from a mathematically rigorous point of view. The paper consists of two parts. The first part, Section 2, contains a survey of integrable systems with known $S$-matrices, and a general approach for solving them, based on constructing wave operators whose kernels are formal eigenfunctions of Bethe-Ansatz type. In the second part, Sections 3 and 4, these ideas are applied to integrable systems connected with pure transmission boundary conditions, resulting in particular in a relativistic dynamics leading to the $S$-matrices of the Federbush model and the Ising model in the scaling limit (references are given in the main text).

More specifically we restrict ourselves in this paper to systems for which a scattering operator exists. In Subsection $2 \mathrm{~A}$ we present a definition of (complete) integrability for these systems that is much stronger than the one that is usually adopted. Subsection $2 \mathrm{~B}$ contains a list of ten (classes of) models that have been shown or are believed to be

* Supported in part by NSF Grant PHY78 23952.

+ Present address: Department of Mathematics, Texas A \& M University, College Station, Texas 77843. 
integrable in this strong sense and for which explicit $S$-matrices are known or have been proposed. We divide these models into two categories. The first category, discussed in Subsection 2C, consists of models whose Hamiltonian is given as an (as a rule formal) operator on a Fock space. (The quantized nonlinear Schrödinger equation can be regarded as a prototype.) For these models one can find formal eigenfunctions by a Bethe-Ansatz. The second category, discussed in Subsection 2D, consists of positive energy relativistic field theories. For these models neither the Hamiltonian nor the space on which it acts are explicitly given. (The $O(3)$ nonlinear $\sigma-$ model can be regarded as a prototype.)

Models from the first category may be rigorously solved by using the formal incoming and outgoing Bethe eigenfunctions to construct two isometric operators $U_{-}$ and $U_{+}$related by $U_{+} S=U_{-}$, where $S$ is the $S$-matrix for the model. These operators (referred to as Bethe transforms) can then be used to define the dynamics rigorously. Subsequently, one proves that the Bethe transforms are the wave operators for this dynamics.

For theories of the second category we argue in Subsection $2 \mathrm{E}$ that a similar Bethe transform solution may exist. We prove this for the Ising and Federbush models in Section 4. For these models we show that the operators $U_{ \pm}$and $S$ are in fact the wave and scattering operators for a large class of dynamics. We reason in Subsection $2 \mathrm{E}$ that the same situation obtains not only for models of the first category, but also for the other field theories, provided they can be solved by the Bethe transform strategy outlined in that subsection. The class of dynamics would contain in particular the even conserved charges of these theories.

In Subsection $2 \mathrm{~F}$ we show that the Bethe transforms $U_{ \pm}$are quantum analogs of the inverse scattering transform in classical integrable field theories. We conjecture that these theories can be cast into a form that is exactly analogous to the approach we propose for quantum systems. We also conjecture that the resulting classical wave and scattering operators are shared by the infinite sequence of even conserved polynomial charges. We show that these conjectures are partly borne out by known results for special cases.

Section 2 ends with Subsection 2G, which contains some other conjectures and problems connected with the results and approach of this paper.

Sections 3 and 4 contain an application of the strategy sketched in Subsection $2 \mathrm{E}$ to the simplest integrable systems with infinitely many degrees of freedom known so far, viz., the continuum Ising and Federbush models. The $S$-matrices of these models suggest a physical picture of $N$ particles on the line moving freely except for independent two-particle collisions, whereupon only transmission occurs and the wave function gets multiplied by an energy-independent phase factor. In this case there are no bound state channels with their ensuing "debauch of indices" and the operators $U_{ \pm}$ (which, from a mathematical point of view, may be regarded as the fundamental objects in these systems) take a particularly simple form.

More particularly, Section 3 contains results on a more general situation of $N$ distinguishable particles. The Bethe transforms $U_{ \pm}$are found by heuristic reasoning, starting from a physical picture as mentioned above. The resulting formal operators 
are subsequently proved to be unitary, and to be the wave operators of a large class of dynamics. Finally, in Section 4 the sectors are pieced together into a Fock space, and a relativistic dynamics leading to the Ising and Federbush model $S$-matrices is constructed. Comments on the relevance of this for the construction of the field theories can be found in Subsections 2D and 2E.

\section{Integrable Systems: An Overview and UNified Approach}

\section{A. Definition of Integrability}

As mentioned in the Introduction, we restrict ourselves in this paper to onedimensional quantum systems with a scattering behavior. More precisely, we shall assume that the dynamics "looks like" a free comparison dynamics in the distant past and future, in the sense that wave operators exist. As we shall presently indicate, this assumption already entails that the system is integrable in the usual sense of there existing a maximal set of mutually commuting independent Hamiltonians. We shall therefore define a much stronger notion of integrability to single out the systems that we intend to discuss in this paper. Roughly speaking, we shall use this term only for systems whose $S$-operator does not couple channels (no particle production and preservation of binding) and conserves the incoming momenta. As a result, integrable systems with infinitely many degrees of freedom decompose into a sequence of $N$-body sectors.

To make this more precise, we shall now consider (as a simple example of such an $N$-body sector) a system of $N$ distinguishable particles on the line that can be described by a wave function from the Hilbert space $\breve{\mathscr{H}} \equiv L^{2}\left(R^{N} ; d x_{1} \cdots d x_{N}\right)$, or equivalently from $\mathscr{H} \equiv L^{2}\left(R^{N} ; d k_{1} \cdots d k_{N}\right)$, where we think of the $x_{i}$ resp. $k_{i}$ as particle positions resp. momenta. Define $J: \mathscr{H} \rightarrow \breve{\mathscr{H}}$ as Fourier transformation and set $\breve{A}=J A J^{-1}$ if $A$ is an operator on $\mathscr{H}$ and vice versa. We assume that the dynamics of the system is given by a self-adjoint Hamiltonian $\check{H}$ on $\check{\mathscr{H}}$ such that the wave operators

$$
\check{W}_{ \pm}=\mathrm{s}_{t \rightarrow \pm \infty} \lim \exp (i \check{H} t) \exp \left(-i \check{H}_{0} t\right)
$$

exist and are unitary. Here, we assume that the free dynamics $H_{0}$ is given by the multiplication operator $\sum_{i=1}^{N} k_{i}{ }^{2}$, so that $\check{H}_{0}$ acts as minus the Laplacean on $\breve{\mathscr{H}}$. In view of what is developed below, it is useful to note that (2.1) is equivalent to existence and unitarity of the operators

$$
U_{ \pm}=s_{t \rightarrow \pm \infty} \lim \exp (i \breve{H} t) J \exp \left(-i H_{0} t\right)
$$

that may be regarded as the wave operators of the system in a two Hilbert space picture of the scattering with identification map $J$ [1]. Clearly,

$$
U_{ \pm}=\check{W}_{ \pm} J
$$


We shall define this system to be integrable if and only if its $S$-operator

$$
S=W_{+}^{*} W_{-}=U_{+}^{-1} U_{-}
$$

on $\mathscr{H}$ commutes with all multiplication operators $A$ of the form

$$
(A f)(k)=\sum_{i=\mathbf{1}}^{N} \alpha\left(k_{i}\right) f(k),
$$

where $\alpha$ is a continuous function on $R$. Equivalently, setting

$$
M_{\sigma}=\left\{k \in R^{N} \mid k_{\sigma(1)}<\cdots<k_{\sigma(N)}\right\},
$$

where $\sigma \in S_{N}$, the symmetric group on $N$ objects, $S$ acts like

$$
(S f)(k)=\sum_{\sigma . \tau \in S_{N}} \chi_{\sigma-1}(k) S_{\sigma \tau}\left(k_{\sigma^{-1}}\right) f\left(k_{\sigma-1}\right) .
$$

Here,

$$
k_{\rho}=\left(k_{\rho(1)}, \ldots, k_{\rho(N)}\right),
$$

$\chi_{\sigma}$ is the characteristic function of $M_{\sigma}$, and the $S_{\sigma \tau}(k)$ are measurable functions defined on $M_{1}$. Note that $(S f)(k)$ need not be defined on the hyperplanes $k_{i}=k_{j}$ since they have measure zero, and note that our definition of $S_{\sigma r}(k)$ is such that in physicist's notation

$$
S_{\sigma \tau}(k)={ }_{\text {out }}\left\langle k_{\sigma} \mid k_{\tau}\right\rangle_{\text {in }}, \quad k \in M_{1} .
$$

A third equivalent way of defining integrability is as follows. Consider the Hilbert space $\ddot{\mathscr{H}} \equiv L^{2}\left(M_{1} ; d k_{1} \cdots d k_{N}\right) \otimes \mathscr{H}_{1 \mathrm{nt}}$, where $\mathscr{H}_{\text {1nt }}=C^{N 1}$. Then one can regard $g \in \mathscr{H}$ as a set of functions $\left\{g_{o}(k)\right\}$ on $M_{1}$, labeled by $\sigma \in S_{N}$. Clearly, $\mathscr{H}$ can be mapped isometrically onto $\tilde{\mathscr{H}}$ by setting

$$
(\text { If })_{\sigma}(k)=f\left(k_{\sigma}\right), \quad k \in M_{1},
$$

so that

$$
\left(I^{-1} g\right)(k)=\sum_{\tau} \chi_{\tau}(k) g_{\tau^{-1}}\left(k_{\tau}\right) .
$$

Regarding $\tilde{\mathscr{H}}$ as a direct integral $\tilde{\mathscr{H}} \simeq \int_{M_{1}}^{\oplus} \mathscr{H}_{\text {int }} d k$ we can define the system as being integrable if and only if $\tilde{S} \equiv I S I^{-1}$ is decomposable with respect to this direct integral, i.e., if and only if there exist measurable functions $S_{\sigma \tau}(k)$ on $M_{1}$ such that

$$
(\tilde{S g})_{\sigma}(k)=\sum_{\tau} S_{\sigma \tau}(k) g_{\tau}(k) .
$$

As indicated by the notation, these functions are equal to the $S_{\sigma \tau}$ in (2.7), which can be easily verified. This third definition is important, since it can be most easily generalized, 
as we shall presently indicate. (The equivalence of the three definitions is a straightforward consequence of the Stone-Weierstrass theorem [2] and a well-known property of constant fiber direct integrals, cf. Ref. [3, Theorem XIII, p. 84].)

One would usually define the quantum system considered above as being completely integrable if there exist $N$ independent mutually commuting operators $\left\{H, H_{2}, \ldots, H_{N}\right\}$. This situation is the quantum analog of the existence of $N$ independent Poisson commuting Hamiltonians if the system is described classically, in which case one defines the classical system as (completely) integrable $[4,5]$. We would like to emphasize that integrability in this sense by no means implies integrability in our sense. Indeed, the system is already integrable in the former sense if the wave operators exist and are unitary. To see this, consider, e.g., the operators

$$
H_{n}{ }^{ \pm} \equiv W_{ \pm} A_{n} W_{ \pm}^{*}, \quad n=1, \ldots, N,
$$

wherc $A_{n}$ is the multiplication operator

$$
\left(A_{n} f\right)(k)=\sum_{i=1}^{N} k_{i}{ }^{n} f(k) .
$$

Then $H_{2}^{ \pm}=H$ by virtue of the intertwining relations, so that we may take $\left\{\mathrm{H}_{1}^{+}\right.$, $\left.H, \ldots, H_{N}^{+}\right\}$or $\left\{H_{1}^{-}, H, \ldots, H_{N}^{-}\right\}$. However, it is to be noted that integrability in our sense does follow if one has

$$
H_{n}{ }^{+}=H_{n}^{-}, \quad n=1, \ldots, N .
$$

Indeed, this is equivalent to

$$
\left[S, A_{n}\right]=0, \quad n=1, \ldots, N,
$$

from which it readily follows that $S$ commutes with all multiplication operators (2.5). Thus the system is integrable in our sense if and only if the incoming "charges" $H_{n}$. equal the outgoing "charges" $H_{n}{ }^{+}$for $n=1, \ldots, N$.

We note in passing that a similar distinction makes sense and would be useful at the classical level. For the systems usually considered in classical mechanics intcgrability in the weak sense is exceptional, since these systems typically have either a compact configuration space, or a non-compact configuration space but attractive (gravitational) interparticle forces. In contrast, for particles on the line with repulsive forces the wave operators are expected to exist except on sets of measure zero, so that such systems are typically integrable in the weak sense. However, again it does not follow from this that the $S$-operator conserves the incoming momenta, which would be the classical analog of the integrability definition given above. For instance, the Calogero system is integrable in this strong sense [5-7], but a perturbation by additional repulsive potentials will in general only be integrable in the weak sense. Note that here too, the (analogs of the) relations (2.15) imply integrability in the strong sense. 
The example we have considered so far may be considered as a prototype for an $N$-body sector of the quantum systems with infinitely many degrees of freedom that are known or believed to have $S$-operators acting only on internal variables. These systems (some of which are listed below) may be described in terms of a Hilbert space with a Fock space structure $\mathscr{F}=\oplus_{N=0}^{\infty} \mathscr{H}_{N}$, i.e., $\mathscr{H}_{0}=C$ corresponds to the vacuum, while $\mathscr{H}_{N}$ consists of $N$-body wave functions. In case no bound states are present, one may regard $\mathscr{H}_{N}$ as the analog of the space $\tilde{\mathscr{H}}$ in the example above. Depending on the system, the $k_{i}$ may be considered as momenta, rapidities, group velocities, etc., while the finite-dimensional space of internal variables $\mathscr{H}_{\text {int }}$ contains the information regarding distinguishability, particle/antiparticle character, negative/ positive effective mass (for magnons), etc. As in the example, the dynamics leaves $\mathscr{H}_{N}$ invariant and $S$ acts only on the fiber $\mathscr{H}_{\text {int }}$. If bound states exist $\mathscr{H}_{\text {int }}$ need not be finite dimensional, but one can split up $\mathscr{H}_{\text {int }}$ into a direct sum of finite-dimensional spaces $\mathscr{H}_{\text {int, } \beta}$, corresponding to certain specified particles and bound states being present. This leads to a direct sum of spaces $\mathscr{H}_{N \beta}$, whereupon the same situation obtains on each $\mathscr{H}_{N \beta}$ separately as in the no bound state case. Thus, also in this case the system reduces to a sequence of $N$-body systems.

\section{B. Integrable Systems with Known S-Matrices}

We shall now try to make this more concrete by listing ten (classes of) quantum systems with infinitely many degrees of freedom for which explicit $S$-operators of this type are known or have been proposed:

1. The Ising model in the scaling limit [8], which is a relativistic field theory of massive neutral bosons, so that $\mathscr{H}_{\text {int }}$ has dimension one.

2. The Federbush model $[9,10]$, a relativistic field theory of two species of massive charged fermions, so that dim $\mathscr{H}_{\text {int }}=4^{N}$.

3. The chiral invariant $S U(n)$ Gross-Neveu model $(n \geqslant 2)$ on the unphysical sector [11]. This is a relativistic field theory describing $n$ massless fermions having positive or negative energy, whence $\operatorname{dim} \mathscr{H}_{\text {int }}=(2 n)^{N}$.

4. The quantized nonlinear Schrödinger equation restricted to the boson sector (or, equivalently, the Laplacean with $\delta$-function pair potentials on symmetric wave functions) [12-15]. This theory describes massive bosons. For one sign of the coupling constant there are no bound states, so that $\operatorname{dim} \mathscr{H}_{\text {int }}=1$, while the other sign leads to one bound state of $n$ particles for any $n>1$, entailing $\operatorname{dim} \mathscr{H}_{\text {int }}=\infty$.

5. The massive Thirring model on the unphysical sector [16-18]. This is a field theory of fermions having positive or negative masses, in which bound states exist. (Note that the $S$-matrices of Ref. [16] and Refs. [17, 18] are different.)

6. The infinite isotropic spin- $\frac{1}{2}$ Heisenberg chain with nearest neighbor interactions in its ground state representation [19-24], describing magnons and their bound states.

7. Model 4 for distinguishable particles [25-27]. In the no bound state case dim $\mathscr{H}_{\text {int }}=N$ ! as in the example above, otherwise $\operatorname{dim} \mathscr{H}_{\text {int }}=\infty$. 
8. The $O(3)$ nonlinear $\sigma$-model [28], a relativistic field theory describing 3 massive neutral bosons without bound states, so that $\operatorname{dim} \mathscr{H}_{\text {int }}=3^{N}$.

9. The $O(n)$ nonlinear $\sigma$-model for $n \geqslant 4$ [28], which is like model 8 , except that its $S$-matrix is qualitatively different (cf. below).

10. The (positive energy) massive Thirring model/sine-Gordon theory [29-31], which is a relativistic field theory of massive charged fermions. It has bound states for one sign of the coupling constant.

For all models listed the $\mathrm{N}$-body amplitudes are built up from two-body amplitudes, corresponding to a soliton picture of the scattering. We have ordered the models in increasing complexity of the two-body amplitudes. For models $1-3$ they are energyindependent phase factors, for models 4-8 rational functions and for model(s) 9 meromorphic functions. For model 10 the two-body amplitudes involving bosons are meromorphic, while the other amplitudes are given by infinite products. It is not obvious to us in what region of the complex plane these products converge, but one of the amplitudes admits an integral representation that defines it as a function analytic in a strip containing the real axis [32-33].

We shall now consider these models in some more detail, pointing out some common features and differences, and discussing strategies for solving them.

\section{C. The Bethe-Ansatz Models}

Models 3-7 on our list have a dynamics that acts on an explicitly given Hilbert space having a Fock space structure $\breve{\mathscr{F}}=\bigoplus_{N=0}^{\infty} \check{\mathscr{H}}_{N}$. Here, the space $\check{\mathscr{H}}_{N}$ is a space of square-integrable wave functions depending on $N$ positions and internal quantum numbers that depend on the model; for instance, for model $7, \breve{\mathscr{H}}_{N}$ equals the space $\breve{\mathscr{H}}$ of the example system above. The spaces $\breve{\mathscr{H}}_{N}$ are manifestly left invariant by the dynamics (for models 3-5 and 7 this holds formally, since the dynamics first needs to be defined). For a mathematically rigorous solution of models $3-5$ and 7 the admitable tour de force of Babbitt and Thomas on model 6 [20-24] may serve as a paradigm, as it actually did in the work of Oxford on model 4 [15]. However, for reasons discussed below, a slightly different approach seems preferable to us. To sketch this approach we shall for simplicity assume from now on that one is dealing with a model without bound states. (To treat theories with bound states in an analogous fashion a large amount of notational machinery needs to be introduced, which would obscure the ideas.) The two main steps are:

A. Defining operators $U_{-}$resp. $U_{+}: \mathscr{F} \rightarrow \mathscr{F}$ in terms of the (suitably normalized) formal incoming and outgoing eigenfunctions of the Hamiltonian, and an operator $J: \mathscr{F} \rightarrow \check{\mathscr{F}}$ that is (roughly speaking) the operator to which $U_{ \pm}$reducc in the casc of no interaction (for models 4 and $7 J$ would be Fourier transformation in the no bound state case; for the bound state case it would also contain the customary channel embedding operators of multi-channel scattering theory [1]); 
B. Proving that $U_{ \pm}$are actually isometric maps from $\mathscr{F}$ onto $\mathscr{F}$, satisfying

$$
U_{+} S=U_{-},
$$

where $S$ is the $S$-operator proposed for the model.

It is to be noted that the original Hamiltonian has not been defined yet; it has only served to define the operators $U_{ \pm}$in terms of its formal eigenfunctions. The reason for deemphasizing the original Hamiltonian is that once steps A and B have been completed the operators $U_{ \pm}$and $S$ can be easily shown to be the wave and scattering operators for a large class $\check{\mathscr{C}}$ of Hamiltonians $\check{H}$ on $\mathscr{F}$ (in a two Hilbert space picture of the scattering with identification map $J[1])$. This class $\check{\mathscr{C}}$ is the transform under $U_{ \pm}$ of a class $\mathscr{C}_{0}$ of free Hamiltonians $H_{0}$ on $\mathscr{F}$, i.e., an element $H_{0} \in \mathscr{C}_{0}$ defines a corresponding element $\check{H} \in \check{\mathscr{C}}$ by

$$
\check{H} U_{ \pm}=U_{ \pm} H_{0} .
$$

The class $\mathscr{C}_{0}$ is a class of self-adjoint multiplication operators $H_{0}$ commuting with $S$ (so that in view of (2.17) the corresponding $\breve{H}$ does not depend on whether one chooses $U_{+}$or $U_{-}$in (2.18)), whose precise definition depends on the model that is being dealt with. (For models 4 and 7 it would contain in particular the multiplication operator $d \Gamma\left(k^{2}\right)$, in which case Eq. (2.18) rigorously defines the formal Hamiltonian one started out from.) Explicit examples can be found in other sections of this paper, but at this point we shall only explain why the relations

$$
U_{ \pm}=\operatorname{s-lim}_{t \rightarrow \pm \infty} \exp (i \breve{H} t) J \exp \left(-i H_{0} t\right)
$$

easily follow for any $H_{0} \in \mathscr{C}_{0}$ and corresponding $\check{H} \in \check{\mathscr{C}}$ once problems $\mathrm{A}$ and $\mathrm{B}$ have been solved. The reason is that the eigenfunctions defining the kernels of $U_{ \pm}$are of Bethe-Ansatz type, i.e., they consist of plane waves and functions that are $C^{\infty}$ in $k$ in the wedges $M_{\sigma}$ defined by Eq. (2.6). This results in stationary phase techniques being tailor-made to prove the relations (2.19). In recent years, these techniques have been elevated to a "high art" by Hörmander [34] (to quote Ref. [1], where the results have been summarized). The point is that once problems A and B have been solved the relations (2.19) follow if one proves that

$$
\left\|f_{ \pm}(t, \cdot)\right\|=\left\|\left(U_{ \pm}-J\right) \exp \left(-i H_{0} t\right) f\right\| \rightarrow 0, \quad \iota \rightarrow \pm \infty,
$$

for $f$ in a total set in $\mathscr{H}_{N}$. The class $\mathscr{C}_{0}$ consists of multiplication operators $H_{0}$ for which the relations $(2.20)$ follow by a stationary phase argument: For these operators and suitable $f$ the functions $f_{ \pm}(t, x)$ vanish in one wedge and rapidly decrease in $x$ and $t$ in the remaining wedges, from which one obtains (2.20) (cf. the proof of Theorem 2 in Subsection 3C).

Summarizing, a picture results of a large class of dynamics $\breve{H}$ on $\breve{F}$ being simultaneously diagonalized by operators $U_{ \pm}$that are the wave operators for all of them, leading to a common $S$-operator. 


\section{D. The Positive Energy Relativistic Field Theories}

Let us now discuss the remaining models 1,2 and $8-10$. These models are positive energy relativistic quantum field theories that have not yet been shown to exist in the sense of axiomatic field theory [35-37], except the sine-Gordon theory [38-39]. (For models 1 and 2 work on this is in progress; partial results for these models can be found in Refs. [40-44].)

However (as we have argued in a previous paper [45]), if the Wightman axioms could be shown to hold and the Haag-Ruelle theory would apply, leading to the $S$ operators proposed for these models, then one is again in the situation described for models 3-7 above, in the sense that the Hilbert space would be a direct sum of $N$-body spaces left invariant by the dynamics. The question then arises whether one can construct relativistic dynamics with this property and leading to the $S$-operator proposed for the model at hand through wave operators that intertwine the interacting representation of the Poincare group and the free representation corresponding to the field theory. As we have explained elsewhere [45], this may facilitate an explicit construction of the fields, since they can then be assumed to act on an explicitly given Fock space, and to transform under an explicitly given representation of the Poincaré group. This is to be contrasted with the implicit construction via removal of cutoffs and application of the reconstruction theorem, which so far has only been shown to work in the superrenormalizable case $[37,46]$, while models $2,8-10$ are renormalizable, but not superrenormalizable (it seems model 1 does not correspond to a Lagrangean field theory).

In a previous paper [45] we presented a scheme for a relativistic particle dynamics that leads by construction to wave operators that intertwine the free and interacting representations of the Poincare group. We studied [47] the question raised above for model 10 on our list along the lines of Subsection $2 \mathrm{~A}$ of that paper. That is, we have attempted to construct an "effective potential" $\lambda(g) V$ such that the reduced Hamiltonian

$$
H_{r}=\cosh \theta+\lambda(g) V
$$

would give rise to the fermion-antifermion transmission and reflection amplitudes [29] and bound state spectrum [48, 49] of the massive Thirring model with bare coupling constant $g$. There are several choices for $V$ that (formally) lead to the correct Born approximation, but we could only calculate the second-order term for one of these. In this case, the transmission is still "correct" (for suitable renormalized coupling $\lambda(g)$ ), but the reflection turns out to be different. In particular it is not crossing symmetric.

The results just discussed are not rigorous and not even completely convincing, so that further research along these lines might be of interest. However, even if one could solve this problem affirmatively on the two-body sector, it is not clear how to proceed on other sectors. In contrast, the approach we are about to sketch considers all sectors, and seems more appropriate and promising for reasons discussed in Subsections $2 \mathrm{E}$ and $2 \mathrm{~F}$. 


\section{E. A Bethe Transform Strategy}

The $S$-operators of all the models on the list have a product structure that corresponds to a physical picture of independent two-body collisions. For models 3-7 this corresponds to the fact that the dynamics can be regarded as a free dynamics except for special boundary conditions at the hyperplanes of coinciding particle positions, and for these models this picture is also clearly reflected in the BetheAnsatz structure of the eigenfunctions that are the kernels of the wave operators $U_{ \pm}$. For the other models the dynamics on the physical positive energy sector is not explicitly given, so that there is no obvious choice for the formal eigenfunctions to define the dynamics rigorously as for the models $3-7$.

However, the structure of the known $S$-operator $S$ on the asymptotic Fock space $\mathscr{F}$ for a model in the former category and the success of the Bethe-Ansatz for a model in the latter category seems to indicate that the following problem may be solved not only for models 1 and 2 (as we show in Section 4), but also for the more interesting field theories 8-10:

Find, by hook or by crook, isometric operators $U_{ \pm}$and a bounded operator $J$, mapping $\mathscr{F}$ onto another Fock space $\mathscr{F}$, with the following properties:

1. The operators $U_{ \pm}$are generalized integral operators whose kernels have a (generalized) Bethe-Ansatz structure, and are related by

$$
U_{+} S=U_{-},
$$

where $S$ is the S-operator of the model in question;

2. The following relations hold,

$$
\mathrm{s}_{t \rightarrow \pm \infty} \lim \left(i \check{H}_{i} t\right) J \exp \left(-i H_{f} t\right)=U_{ \pm},
$$

where $H_{f}$ is the time translation generator of the free representation $U_{f}(a, \Lambda)$ of the Poincaré group on $\mathscr{F}$, and $\breve{H}_{i}$ the time translation generator of the interacting representation $\breve{U}_{i}(a, \Lambda)$ on $\breve{F}$, defined by

$$
\check{U}_{i}(a, \Lambda) U_{ \pm}=U_{ \pm} U_{f}(a, \Lambda) .
$$

(Note that $\check{U}_{i}$ is well defined since $S$ is Lorentz invariant, i.e., commutes with $U_{f}(a, \Lambda)$.)

If one could construct Bethe transforms $U_{ \pm}$and a comparison map $J$ as above, it seems plausible that there exists again a whole class of dynamics (containing in particular the even conserved charges of the model) for which $U_{ \pm}$and $S$ are the wave and scattering operators. Among these dynamics the dynamics generated by $\check{H}_{i}$ above is singled out by physical considerations, viz., the requirement of relativistic invariance.

Recently, a number of $S$-operators having a structure similar to those of models 
1-10 were constructed, based on requirements of invariance with respect to the Poincaré group and an internal symmetry group, and on imposition of crossing and "factorization" relations [50]. It seems these $S$-operators do not correspond to Lagrangean field theories. Here too, the fact that this highly nontrivial set of restrictions admits (almost unique) solutions leads us to believe that a bounded operator $J$ and isometric operators $U_{ \pm}$as above exist, whose isometry hinges on the severe restrictions that have been imposed on the scattering amplitudes. As for models 8-10, there does not seem to be an obvious choice for these operators. In particular, though the space they should be defined on is given (up to unitary maps like I in Eq. (2.10)), there does not seem to be an obvious choice for the space they should map to (in contrast to the models of Subsection 2C).

It is to be emphasized that a construction of such operators only gives an affirmative answer to the question whether a relativistic dynamics exists that leads to the $S$-matrix at hand. Though this is a necessary condition for the existence of a Wightman field theory leading to this $S$-matrix via the Haag-Ruelle theory, solving the problem above does not seem to have immediate consequences for the existence of field operators or local charges defined in terms of them. As regards local charges, this may already be seen for model 4: A rigorous definition of interacting field operators is trivial in this case once problems A and B in Subsection $2 \mathrm{C}$ have been solved. However, for $n \geqslant 6$ the conserved charges $U_{ \pm} d \Gamma\left(k^{n}\right) U_{ \pm}^{-1}$ on $\check{F}$ do not admit a representation as integrals of normally ordered polynomial expressions in terms of the time zero field operators, as suggested by the classical conservation laws [51]. Moreover, though such expressions exist for $n=4,5$, they disagree with their classical counterparts. This has been shown by Oxford [15], refuting a conjecture made in Ref. [52]. Similarly, for models 8-10 the classical charges have quantum analogs that can be shown to be conserved in perturbation theory (cf. Refs. [53,54] and references given therein), but it is not at all clear that they are expressible in the nonperturbative field operators (assuming they exist). In any case, the physical significance of this property is unclear. It should also be noted that the charges do not belong to the quasilocal algebra (i.e., they have a global character), even if they admit local densities.

\section{F. Comparison with the Inverse Scattering Transform}

Considered as classical field theories the models 4 and 10 have been shown to be integrable Hamiltonian systems in papers [51,55-58] patterned after the pioneering work of Zakharov and Faddeev on the Korteweg-deVries equation [59]. (The results of Refs. [60,61] indicate that this also holds for models 3, 8 and 9.) In order to compare the classical and quantum systems we shall briefly summarize these results: Through the inverse scattering formalism [62-64] one can obtain a one-one correspondence between fixed time field configurations $\mathscr{F}$ for the respective wave equations and a set of scattering data for an associated linear eigenvalue problem, and these data evolve essentially linearly if the field evolves according to the nonlinear wave equation. Suitable functions of the scattering data can be used as canonical coordinates on a symplectic manifold $\mathscr{F}$ that is mapped onto $\mathscr{F}$ by a canonical transformation $U$ 
that is essentially the inverse scattering transform. There exists a Hamiltonian $H_{0}$ on $\mathscr{F}$ that depends only on generalized momenta and that is such that the linear flow on $\mathscr{F}$ generated by it is mapped by $U$ onto the nonlinear evolution on $\mathscr{F}$, which can be regarded as the flow generated by a Hamiltonian $\check{H}$ on $\mathscr{F}$. Thus one has

$$
\exp (t \check{H}) U=U \exp \left(t H_{0}\right)
$$

or, equivalently, since $U$ is canonical

$$
\check{H} \circ U=H_{0} .
$$

Moreover, the same situation obtains for the nonlinear evolutions generated by the infinite set of polynomial charges $\breve{H}_{n}$, showing on one hand that all $\breve{H}_{n}$ are conserved under the evolution generated by any one of them, and on the other hand that they all have the same radiation and $N$-soliton sectors in $\mathscr{F}$.

As we have already suggested by our notation in the preceding paragraph, the unitary operators $U_{-}$and $U_{+}$for models 3-7 are in many respects the quantum analogs of the canonical transformation $U$. Indeed, the quantum analog of a fixed time field configuration is a vector in $\mathscr{F}$, which can be written as a superposition of its $N$-body sector projections. The quantum-mechanical Hamiltonians $\breve{H}_{n}$ generate oneparameter unitary groups that leave the sectors invariant, and are simultaneously diagonalized by $U_{ \pm}$as multiplication operators $H_{n, 0}$ on $\mathscr{F}$. Furthermore, the inverse scattering transform can be regarded as a generalization of Fourier analysis to nonlinear evolution equations [64], while the Bethe transforms can be regarded (in view of the structure of their kernels in terms of plane waves and phase factors) as generalizations of the Fourier transformation to interacting quantum systems.

However, in order to point out and conjecture further analogies between classical and quantum integrable systems it is convenient to first signal a physical difference between the quantum operators $U_{ \pm}$and the classical map $U$, viz., the fact that $U_{ \pm}$are the wave operators (2.19) for the quantum system, while $U$ is simply a diagonalizing map that contains no immediate information concerning the scattering behavior of the classical system. Correspondingly, one should think of the quantum mechanical $H_{0}$ and $\mathscr{F}$ as a free dynamics acting on a space of free particles that is being compared in the distant past and future with an interacting dynamics $\breve{H}$ acting on $\mathscr{F}$ via a comparison operator $J$ mapping $\mathscr{F}$ onto $\mathscr{F}$. This operator $J$ is in general not unitary (in particular it is not if bound state sectors occur); it is defined so as to take into account that the interacting dynamics admits freely evolving one-body states. (The reader unfamiliar with these ideas could consult Ref. [1], where they are worked out in detail for non-relativistic multi-channel scattering, magnon scattering and the Haag-Ruelle theory.) In contrast, the classical $H_{0}$ and $\mathscr{F}$ are simply canonical transforms of $\check{H}$ and $\mathscr{F}$.

It seems very likely to us that one can cast the classical integrable systems mentioned above into a form that is the exact analog of the quantum situation. That is, it should be possible to give a formulation along the following lines: The classical manifold $F$ 
and evolutions $H_{n, 0}$ are now regarded as describing freely evolving radiation and linear superpositions of freely evolving solitons. There exist two canonical transformations $U_{-}$and $U_{+}$mapping $\mathscr{F}$ onto the set $\mathscr{F}$ of fixed time field configurations for the nonlinear wave equation in question, connected by

$$
U_{+} S=U_{-},
$$

where $S$ is a canonical map on $\mathscr{F}$. There exists a (non-canonical) map $J: \mathscr{F} \rightarrow \mathscr{F}$ such that

$$
\exp \left(-t \breve{H}_{n}\right) J \exp \left(t H_{0, n}\right) \stackrel{t \rightarrow \pm \infty}{\longrightarrow} U_{ \pm}
$$

(in a suitable topology), so that $U_{ \pm}$and $S$ are the wave and scattering maps for all of the polynomial conserved charges $\breve{H}_{n}$ of the theory. As a consequence the scattering map $S$ commutes with all of the free Hamiltonians $H_{0, n}$ on $\mathscr{F}$, and therefore leaves the generalized momenta invariant.

This picture is at least partly borne out by results in the literature: For the nonlinear Schrödinger equation canonical maps $U_{ \pm}$and $S$ satisfying (2.27) exist, and $S$ describes the scattering on $\mathscr{F}$ [65]. In the case of the Korteweg-deVries equation the thorough analysis of Ref. [66], combined with the results of Ref. [59], is easily seen to imply that the shifts in the soliton positions are the same for the infinite sequence of higher order flows; one only needs to replace $\kappa_{p}{ }^{2}$ in Eq. (3.33) in Ref. [66] by $\kappa_{p}^{2 n}$ and then follow the argument given there. More generally, a similar argument presumably works for the $\mathrm{N}$-soliton solutions of other wave cquations, showing that on the solition sectors the same phase shifts occur for all the charges. In the case of the $\mathrm{KdV}$ equation the phase shifts can be easily "translated" to $\mathscr{F}$, since they amount to shifting the eigenfunctions by virtue of Eq. (3.13) in Ref. [66]. Hence, the norming constants get shifted, and this leads to shifts of the generalized positions in $\mathscr{F}$. Again a similar argument should work for other equations, and presumably gives rise to a canonical map $S$ on the soliton sectors in $\mathscr{F}$, as in Ref. [65]. For the radiation the situation is much less clear, in particular as regards the choice of $J$ in Eq. (2.28), the limit involved there, and the question whether the same scattering occurs for all the charges. (It is interesting to note that the quantum theory is simpler in this respect, since (pictorially speaking) the radiation turns into solitons upon quantization.) A further analysis of these matters seems to us of considerable interest, but is beyond the scope of this paper.

It is also of interest to consider the converse question, viz., whether a natural quantum analog of the classical map $U$ exists. This question can be answered affirmatively for the $N$ free particle sectors of models 4 and 5: For model 4 the space $\mathscr{H}_{\text {int }}$ corresponding to this sector is one-dimensional; for model 5 this dimension is $2^{N}$, but the $S$-operator is diagonal in the natural basis, since only transmissions occur. Therefore, there is a natural definition of the square root of $S$. On these sectors the operator

$$
U \equiv U_{+} S^{1 / 2}=U_{-} S^{-1 / 2}
$$

can be regarded as the quantum analog of $U$. Indeed, at the classical level there exists 
a canonical transformation $T$ whose square is the canonical map $S$ for model 4 , as can be seen from the results of Ref. [65], and we expect that this is also true for other models. The natural classical analogs of the operators $U_{ \pm}$are then the maps

$$
U_{ \pm}=U T^{\mp 1} \text {. }
$$

This association is further confirmed by recent work of Thacker and Wilkinson [67] and Honerkamp et al. [68]: these authors show that a Jost function of the classical inverse scattering problem can be formally quantized so as to give rise to the Bethe eigenfunctions that constitute the kernel of $U$, while other Jost functions give the kernels of $U_{ \pm}$. It seems less clear how to define a quantum analog of $U$ for the other sectors of models 4 and 5 , and for the other models on the list.

We would like to close this subsection by briefly discussing one more issue, viz., the question whether the $N$-soliton phase shifts can arise as the scattering map of an $N$ particle Hamiltonian. We have studied this for the sine-Gordon equation on the twosoliton sector [47]. If one soliton has rapidity $\theta_{1}$, and the other one rapidity $\theta_{2}<\theta_{1}$ one easily verifies using the two-soliton solution [69] that the shifts are $-\left(\cosh \theta_{1}\right)^{-1}$ $\ln \tan h^{2}\left(\theta_{2}-\theta_{1}\right) / 2$ resp. $\left(\cosh \theta_{2}\right)^{-1} \ln \tan h^{2}\left(\theta_{2}-\theta_{1}\right) / 2$. Formally transporting this to the two-soliton sector in the symplectic manifold $\mathscr{F}$ leads, up to a constant, to the same shifts of the generalized positions except that the Lorentz-Fitzgerald contraction factors do not occur; the generalized momenta minus constants are proportional to the rapidities. This can be seen from the results of Ref. [55], which, unfortunately, have not been formulated in this manifestly Lorentz invariant fashion. In a previous paper [45] we studied classical dynamics that give rise to Lorentz invariant $S$-matrices by construction. In particular, we solved the inverse scattering problem for Hamiltonians of the form $\cosh \theta+V(x)$ in Subsection $2 \mathrm{~B}$ of that paper. It is not hard to see from the results obtained there that the sine-Gordon phase shift cannot arise as the $S$-matrix of a potential in the class considered there. Of course, this does not exclude that other relativistic dynamics exist leading to this phase shift. However, it is to be noted that even if the classical sine-Gordon theory could be formulated along the lines sketched above, there is no guarantee that such a Hamiltonian dynamics exists on the soliton sectors in $\mathscr{F}$, since it is unlikely that one can choose $J$ in Eq. (2.28) to be canonical on the soliton sectors (if $J$ were canonical, the dynamics $H=\check{H} \circ J$ on $\mathscr{F}$ would have the required property). This is completely analogous to the situation in the quantum case: The corresponding quantum problem discussed at the end of Subsection 2D may not have a solution if $J$ in Eq. (2.23) cannot be chosen to be isometric. And even if $J$ is isometric it may not be possible to write the interacting dynamics as a sum of the free dynamics and a perturbation. This is actually the case for both the continuum Ising model and the Federbush model, as we shall show in Section 4.

\section{G. A List of Problems and Conjectures}

We have already mentioned several problems and conjectures in connection with the approach sketched above. We shall finish this section by presenting some more. 
We divide this material into eight categories whose headings we shall now list for ease of reference.

1. relations between models

2. finding $U_{ \pm}$

3. product structurc of $S$

4. boundary conditions
5. invariance principle

6. associated field theory

7. classification

8. higher dimensions

1. Relations between models. Work by various authors [17, 18, 70-73] suggests that:

$\alpha$. The anisotropic infinite Heisenberg chain in its ground state representation is an integrable system in our sense;

$\beta$. Its continuum limit is model 10 on our list.

Work by Dashen et al. [48] suggests that

$\gamma$. The limit of model 10 as $\gamma^{\prime} \rightarrow 0$ (using their notation) and $\bar{c} \rightarrow \infty(\bar{c} \equiv$ the speed of light), with $\gamma^{\prime} \bar{c} \equiv-8 c$ fixed, is model 4 with negative coupling constant $c$ (using the notation of Refs. [12-15]).

$\alpha, \beta$ and $\gamma$ are presumably very hard problems to prove, so it is useful to raise analogous questions that may be simpler to answer. In this vein we have considered the isotropic Heisenberg chain (model 6), for which problem $\alpha$ has been solved by Babbitt and Thomas [20-24]. In this case the continuum limit can be proved to be a gas of free nonrelativistic bosons [74]. Conversely, one may ask whether integrable infinite chain systems exist whose continuum limit equals models 1-5 resp. $7-10$. The continuum limit and the limit in problem $\gamma$ we have in mind is strong convergence of the Bethe transforms $U_{ \pm}$(assuming they can be constructed), which would presumably easily imply (like in Ref. [74]) that the dynamics converge in the strong resolvent sense. It would also imply that the "boson $S$-operator" of the sine-Gordon theory [30-31] strongly converges to the $S$-operator of the attractive nonlinear Schrödinger equation [15] in this limit. It is straightforward to verify that this indeed happens, so that this necessary condition is satisfied. The disappearance of the soliton/antisoliton sectors for problem $\gamma$ in this limit would be analogous to the disappearance of the bound magnon sectors in the continuum limit of the isotropic Heisenberg chain [74].

2. Finding $U_{+}$. For model 10 and for the positive energy version of model 3 it is not clear whether Bethe transforms $U_{ \pm}$(as defined in Subsection $2 \mathrm{E}$ ) exist. There are interesting papers by Bergknoff and Thacker [17, 18] and by Andrei and Lowenstein [11], showing that the bound state spectrum of these theorics can be formally obtaincd by using results for these models on the unphysical sector (i.e., models 3 and 5 on our list). There is another paper by Honerkamp and Weber [75] showing that one of Zamolodchikov's [29] model 10 scattering amplitudes may be recovered in this 
fashion. We ask: can this method ("third quantization"?) be used to make an educated guess for what $U_{ \pm}$should look like?

3. Product structure of $S$. We have defined integrability of a system in terms of its $S$-operator satisfying (a generalization of) Eq. (2.12) but in all cases known to us $S$ operators of this type actually have a product structure, as described above. Thus, the problem arises whether this is a consequence of Eq. (2.12), and if not, to construct a counterexample. One can also ask this question at the level of axiomatic field theory: If an $S$-operator of the form (2.12) results from the Haag-Ruelle theory applied to a Wightman field theory, does it necessarily have a product structure as encountered in models 1, 2, 8-10? An analogous question in axiomatic $S$-matrix theory has been answered affirmatively by Iagolnitzer [76, 77], but here one makes strong factorization assumptions on the $S$-matrix at the outset. As far as we know these assumptions have not been shown to follow from the Haag-Ruelle theory. However, since they hold in perturbation theory, it is plausible that the answer to the question posed is affirmative. Can this be proved?

4. Boundary conditions. The models $1-5$ and 7 on our list have $S$-operators that admit dynamics given by differential operators whose domain consists of wave functions satisfying certain boundary conditions on the hyperplanes $x_{i}=x_{j}$ in $R^{N}$. (This also holds, mutatis mutandis, for model 6.) Is it possible to construct other integrable systems by specifying other boundary conditions? For instance, the operator $(d / d x)^{2}$ restricted to $C_{0}^{\infty}(R \backslash\{0\})$ admits a four-parameter family of selfadjoint extensions that are specified by boundary conditions at the origin. Some of these extensions correspond to models $1,2,4$ and 7. The other extensions can in principle be used to construct operators $U_{ \pm}$of Bethe-Ansatz type. Some extensions lead to transmission and reflection coefficients that do not satisfy the so-called "factorization equations" [28], which implies there are several ways of specifying $U_{+}$. Does the lack of factorization entail that such $U_{ \pm}$are not isometric?

5. Invariance principle. The fact that $U_{ \pm}$and $S$ are the wave and scattering operators for a large class of dynamics is reminiscent of the well-known invariance principle for the wave operators [1], but it is not implied by it, since none of the operators $H_{0} \in \mathscr{C}_{0}$ is a function of any other one. However, from the results of the next two sections it is clear that in simple cases the operators $d \Gamma\left(k^{2 n}\right), n=1,2, \ldots$, will belong to $\mathscr{C}_{0}$, and the invariance principle does apply to the "arguments" of $d \Gamma$. Therefore, this fact seems to hint at the existence of a more general invariance principle that would, e.g., imply the following:

Assume

$$
\operatorname{sim}_{t \rightarrow \pm \infty} \exp \left(i H_{1} t\right) \Gamma\left(\exp \left(-i k^{2} t\right)\right)=W_{ \pm}
$$

is a unitary operator on $\mathscr{F}=\oplus_{\mathrm{N}=0}^{\infty} L^{2}\left(R^{N} ; d k_{1} \cdots d k_{N}\right)$ for some self-adjoint $H_{1}$ that leaves the sectors in $\mathscr{F}$ invariant. Set

$$
{H_{n}}^{ \pm} \equiv W_{ \pm} d \Gamma\left(k^{2 n}\right) W_{ \pm}^{*}, \quad n=1,2, \ldots
$$


If

$$
H_{n}{ }^{+}=H_{n}{ }^{-}=H_{n}, \quad n=2,3, \ldots
$$

or, equivalently,

$$
\left[S, d \Gamma\left(k^{2 n}\right)\right]=0, \quad n=2,3, \ldots
$$

then

$$
\operatorname{s}_{t \rightarrow \pm \infty} \operatorname{limp}\left(i H_{n} t\right) \Gamma\left(\exp \left(-i k^{2 n} t\right)\right)=W_{ \pm}, \quad n=1,2, \ldots .
$$

The reader familiar with the usual invariance principle will have no trouble in formulating various versions of such a more general invariance principle (each of which may be false!) in abstract operator language. (It is also conceivable that if (2.33)(2.34) hold only for some $n>1$ it already follows that (2.35) holds for these $n$.) This seems an interesting problem in quantum scattering theory, whose solution might shed more light on integrable systems.

A related question is whether the wave and scattering operator invariance discussed in this paper also holds for integrable (in our sense) $N$-particle systems that we have not considered. For instance, in the classical Calogero model one finds that the scattering can be pictured as a sequence of billiard ball collisions, i.e., no phase shifts occur [5-7]. The results of this paper lead us to conjecture that this is also true for the $N-1$ higher order conserved Hamiltonians.

6. Associated field theory. Suppose one has constructed an integrable $N$-body system with a relativistic positive energy dynamics leading to a Lorentz invariant $S$ operator $S_{N}$. Is it possible to construct a Wightman field theory (with fields that do not necessarily correspond to fields in a formal interaction Lagrangean), whose Haag-Ruelle $S$-operator coincides with $S_{N}$ on the $N$-body sector? This question is in particular relevant in connection with Ref. [50] quoted above, but is also of obvious interest for models $1,2,8-10$.

7. Classification. Is it possible to classify integrable quantum systems of the type we are considering? This obvious question is presumably on the same level of difficulty as its classical analog, to which even after fifteen years only partial answers have been found [78-79]. In view of our definition this amounts to characterizing the $S$-operators that may arise. For instance, are there $N$-body dynamics with $S$-operator $\exp (i \phi N(N-1) / 2) \quad(\phi \neq n \pi, n \in Z)$ ? Note that from the point of view we have sketched above, this is tantamount to asking whether isometric operators $U_{ \pm}$exist that satisfy (2.17) and that are the wave operators of certain dynamics in the sense of Eq. (2.19).

8. Higher dimensions. We have restricted ourselves to one-dimensional systems. There seems to be no obvious choice for a higher-dimensional generalization of the integrability definition given in Subsection 2A. However, one may ask: Are there higher-dimensional systems with analogous properties? A simple and explicit question along these lines is: Are there $N$-body dynamics on $L^{2}\left(R^{3 N}\right)$ with $S$-operator 
$(-)^{N(N-1) / 2}$ ? In this connection it is to be noted that $S$-operators of the latter type fail to satisfy the usual cluster properties $[1,80]$ known to hold (under certain assumptions) in three-dimensional nonrelativistic quantum mechanics [81] and field theory [82]. Another relevant result is the so-called Coleman-Mandula theorem [83-85], showing (roughly speaking) that symmetries of higher-dimensional field theories with sufficient interaction are always products of internal and Poincare group charges. This last question is of particular physical importance, since there exist certain formal analogies between non-abelian gauge theories in four space-time dimensions and integrable field theories in two space-time dimensions.

\section{An Integrable $N$-Particle System Associated with Pure Transmission BOUNDARY CONDITIONS}

As mentioned before, the continuum Ising model describes neutral bosons, while the Federbush model describes two species of charged fermions. The $S$-matrix for two Ising bosons is -1 , while the $S$-matrix for two Federbush fermions is one if they are of the same species, $\exp ( \pm i \phi)$ if they are of different species. The sign in the phase depends on whether the species I fermion came from the left or from the right with respect to the species II fermion and on the charges. In both cases the $N$-body $S$-matrix is a product of the $N(N-1) / 2$ two-body $S$-matrices. Instead of restricting ourselves to these two models and dealing with them separately as sketched in Subsection 2E, it is in fact more convenient and illuminating to consider a more general system of $N$ distinguishable particles in which a similar scattering occurs. This system is of some interest in its own right, and the results obtained can easily be translated to the $N$-body sectors of the field theories. Moreover, the proofs of the relevant results for this system are actually simpler and more transparent than their analogs for the Federbush model, and clearly bring out the general features discussed in the preceding section.

To describe the system we shall use without further comment the notation already introduced in Subsection 2A for the example discussed there. The physical picture of the scattering in our system is as follows. In the two-particle case, particles 1 and 2 move along the line till they hit each other, whereupon only transmission occurs. Their joint wave function gets multiplied by $\exp (i \phi)$ resp. $\exp (-i \phi)$ if the order (i.e., left, right) changes from 12 to 21 , resp. 21 to 12 . For general $N$ the resulting $N$-soliton picture is then that of $N(N-1) / 2$ independent two particle collisions, after which the wave function is multiplied by a phase $\exp (i k \phi)$, where $k$ ranges from $-N(N-1) / 2$ $(N \cdots 21 \rightarrow 12 \cdots N)$ to $N(N-1) / 2(12 \cdots N \rightarrow N \cdots 21)$. A moment's thought now shows that the corresponding $S$-operator on $\mathscr{H}$ is the unitary multiplication operator

$$
(S f)(k)=\exp \left[i \phi \sum_{1 \leqslant i<j \leqslant N} \epsilon\left(k_{i}-k_{j}\right)\right] f(k)
$$

where $\epsilon(x)= \pm 1$ for $x \gtrless 0$.

In keeping with the ideas in Subsection 2E we shall now "guess" the corresponding 
Bethe transforms $U_{ \pm}$, and subsequently prove them to be unitary, to satisfy (2.22), and to be equal to the wave operators for a large class of dynamics. Subsection $3 \mathrm{~A}$ contains the physical argument leading to the expressions for $U_{ \pm}$, Subsection $3 \mathrm{~B}$ a precise statement of the results, and Subsection $3 \mathrm{C}$ the proofs.

\section{A. Construction of the Bethe Transforms}

We want to define two (generalized) integral operators mapping $\mathscr{H}$ onto $\check{\mathscr{H}}$, whose kernels $U_{-}(x, k)$ resp. $U_{+}(x, k)$ can be regarded as formal incoming resp. outgoing eigenfunctions of the dynamics that they define and whose wave operators they should be. Moreover, we would like $U_{ \pm}(x, k)$ to have a Bethe-Ansatz structure, which corresponds to a physical picture of the system evolving freely in the wedges $M_{\sigma}$ (cf. Eq. (2.6)) until a hyperplane $x_{i}=x_{j}$ in $R^{N}$ is reached. We shall now combine this with the scattering picture sketched above to find the kernels $U_{ \pm}(x, k)$.

Let us first consider $U_{-}(x, k)$ for $k$ in the wedge $M_{\tau}$. Denote the permutation $(12 \cdots N) \rightarrow(N \cdots 21)$ by $r$. Since $k_{\tau r(1)}>\cdots>k_{r r(N)}$ the particles were in the order $\operatorname{\tau r}(1)<\cdots<\tau r(N)$ in the distant past, i.e., their positions $x=\left(x_{1}, \ldots, x_{N}\right)$ were in the wedge $M_{\tau r}$. Now $U_{-}(x, k)$ is an incoming wave function, so that $\chi_{\tau r}(x) U_{-}(x, k)$ $\chi_{\tau}(k)$ should contain a free plane wave $\exp (i k x)$. But the particles cannot get back to $M_{\tau r}$ in the course of their evolution, since only transmissions occur. Hence we can take $\chi_{r r} U_{-} \chi_{r}=\exp (i k x)$. The particles can get to any other wedge $M_{\sigma}$ while keeping their momenta, and they evolve freely in $M_{\sigma}$ until a bounding hyperplane is reached. Thus one has $\chi_{\sigma} U_{-} \chi_{\tau}=C_{\sigma \tau} \exp (i k x)$, where the phase factor $C_{\sigma \tau}$ is determined by the transmissions that occur to get from $M_{\tau r}$ to $M_{\sigma}$. To obtain $C_{\sigma \tau}$ we note that one picks up a phase $\exp \left(i \phi \epsilon\left(k_{i}-k_{j}\right)\right)$ only for those pairs $i<j$ whose order changes in this process, i.e., such that $\left[\sigma^{-1}(i)-\sigma^{-1}(j)\right]\left[r \tau^{-1}(i)-r \tau^{-1}(j)\right]<0$. Thus, setting

$$
\theta_{\kappa \nu}(i, j)=\theta\left(\left[\kappa^{-1}(i)-\kappa^{-1}(j)\right]\left[\rho^{-1}(j)-\rho^{-1}(i)\right]\right),
$$

where $\theta(x)={ }_{0}^{1}$ for $x \gtrless 0$, our argument shows that one should define $U_{-}$as

$$
\begin{aligned}
\left(U_{-} f\right)(x)= & (2 \pi)^{-N / 2} \sum_{\sigma, \tau} \chi_{\sigma}(x) \int d k \exp \left[i \phi \sum_{i<i} \theta_{\sigma, \tau r}(i, j) \epsilon\left(k_{i}-k_{j}\right)\right] \\
& \cdot \exp (i k x) \chi_{\tau}(k) f(k) .
\end{aligned}
$$

Similarly, one should have $\chi_{\tau} U_{+} \chi_{\tau}=\exp (i k x)$, since the particles will be in the wedge $M_{\tau}$ in the distant future if their momenta are in $M_{\tau}$. In this case, the phase $\bar{C}_{\sigma \tau}$ in $\chi_{\sigma} U_{+} \chi_{\tau}=\bar{C}_{\sigma \tau} \exp (i k x)$ follows from the requirement that the particles arrive without phase in going from $M_{\sigma}$ to $M_{\tau}$. As a result we define

$$
\begin{aligned}
\left(U_{+} f\right)(x)= & (2 \pi)^{-N / 2} \sum_{\sigma, \tau} \chi_{\sigma}(x) \int d k \exp \left[-i \phi \sum_{i<j} \theta_{\sigma \tau}(i, j) \epsilon\left(k_{i}-k_{j}\right)\right] \\
& \cdot \exp (i k x) \chi_{\tau}(k) f(k) .
\end{aligned}
$$




\section{B. The Results}

We are now in a position to state the first theorem.

THEOREM 1. The operators $U_{ \pm}$are unitary maps from $\mathscr{H}$ onto $\check{\mathscr{H}}$, satisfying

$$
U_{+} S=U_{-} .
$$

Using this result we shall now define the class of dynamics for which $U_{ \pm}$and $S$ will turn out to be the wave and scattering operators (Theorem 2). To this purpose we introduce the class $\mathscr{C}_{0}$ of free Hamiltonians of the form

$$
\left(H_{0} f\right)(k)=\sum_{i=1}^{N} h\left(k_{i}\right) f(k) .
$$

Here, $h(k)$ is a real-valued $C^{\infty}$ function on $R$ such that $h^{\prime}(k)$ is strictly increasing in $k$ (e.g., $k^{2 n}, m \cosh n k(n=1,2, \ldots),\left(k^{2}+m^{2}\right)^{1 / 2}$, etc.). We can now define a class $\check{\mathscr{C}}$ of corresponding perturbed dynamics $\breve{H}$ by setting

$$
\check{H} U_{\ddagger} \equiv U_{ \pm} H_{0} .
$$

Note that $\breve{H}$ is well defined since $U_{ \pm}$are unitary and related by Eq. (3.5). We can now state the second result.

THEOREM 2. For any $H_{0} \in \mathscr{C}_{0}$ and corresponding $\check{H} \in \check{\mathscr{C}}$ one has

$$
\operatorname{s-lim}_{t \rightarrow \pm \infty} \exp (i \breve{H} t) J \exp \left(-i H_{0} t\right)=U_{ \pm},
$$

where

$$
(J f)(x) \equiv(2 \pi)^{-N / 2} \int d k \exp (i k x) f(k) .
$$

(Of course, the integral here and in Eqs. (3.3)-(3.4) stands for a limit in the mean.) Note that in this case it is not necessary to use two Hilbert space scattering theory, since $J$ is actually unitary. (That is, we could have formulated Theorem 2 in terms of the wave operators $\check{W}_{ \pm}=U_{ \pm} J^{-1}$ on $\breve{\mathscr{H}}$.) However, as mentioned before this will not be generally true for other models, so that we prefer the above form.

\section{C. The Proofs}

Proof of Theorem 1. We shall show the unitarity of $U_{+}$. The proof for $U_{-}$is similar. First, we prove that $U_{+}$is isometric. To this end we define

$$
\mathscr{D}=\left\{f \in \mathscr{H} \mid f \in C_{0}{ }^{\infty} \text { and } \operatorname{supp} f \in M_{o} \text { for some } \sigma\right\} .
$$

Since $\mathscr{D}$ is total in $\mathscr{H}$ the isometry of $U_{+}$will follow if we show that

$$
\left(U_{+} f, U_{+} g\right)=(f, g) \quad \forall f, g \in \mathscr{D} .
$$


To prove this, let $\operatorname{supp} f \subset M_{\tau_{1}}$, supp $g \subset M_{\tau_{2}}$. Because of the relation

$$
\epsilon\left(k_{i}-k_{j}\right)=\epsilon\left(\tau^{-1}(i)-\tau^{-1}(j)\right), \quad k \in M_{\tau},
$$

we then have

$$
\begin{aligned}
\left(U_{+} f, U_{+} g\right)= & (2 \pi)^{-N} \sum_{\sigma} \int_{M_{\sigma}} d x \exp \left[i \phi \sum_{i<j} F_{o \tau_{1} \tau_{2}}(i, j)\right] \\
& \cdot \int_{M_{\tau_{1}}} d k \exp (-i k x) \bar{f}(k) \int_{M_{\tau_{2}}} d k^{\prime} \exp \left(i k^{\prime} x\right) g\left(k^{\prime}\right),
\end{aligned}
$$

where we have set

$$
F_{\sigma \tau_{1} \tau_{2}}(i, j) \equiv \theta_{\sigma \tau_{1}}(i, j) \epsilon\left(\tau_{1}^{-1}(i)-\tau_{1}^{-1}(j)\right)-\theta_{\sigma \tau_{2}}(i, j) \epsilon\left(\tau_{2}^{-1}(i)-\tau_{2}^{-1}(j)\right) .
$$

But if $n, m_{1}$ and $m_{2}$ are non-zero integers, one readily verifies that

$$
\theta\left(n m_{1}\right) \epsilon\left(m_{1}\right)-\theta\left(n m_{2}\right) \epsilon\left(m_{2}\right)=\theta\left(m_{1}\right)-\theta\left(m_{2}\right) .
$$

Using this to rewrite $F$ and inserting in (3.13) we obtain by virtue of Parseval's relation

$$
\left(U_{+} f, U_{+} g\right)=\exp \left[i \phi \sum_{i<j}\left[\theta\left(\tau_{1}^{-1}(i)-\tau_{1}^{-1}(j)\right)-\theta\left(\tau_{2}^{-1}(i)-\tau_{2}^{-1}(j)\right)\right]\right](f, g) .
$$

But $(f, g)=0$ if $\tau_{1} \neq \tau_{2}$, so that (3.11) follows. Thus $U_{+}$is isometric, as claimed.

Since $U_{+}$is isometric, its unitarity will follow if we prove that $U^{*}$ is isometric. To show this, we observe that for $\psi \in \ddot{\mathscr{H}} \cap C_{0}{ }^{\infty}$ one has

$$
\begin{aligned}
\left(U_{+}^{*} \psi\right)(k)= & (2 \pi)^{-N / 2} \sum_{\tau, \sigma} \chi_{\tau}(k) \exp \left[i \phi \sum_{i<j} \theta_{\sigma \tau}(i, j) \epsilon\left(k_{i}-k_{j}\right)\right] \\
& \cdot \int d x \exp (-i k x) \chi_{\sigma}(x) \psi(x) .
\end{aligned}
$$

From this the isometry of $U_{+}^{*}$ follows by an argument analogous to the one that proved isometry of $U_{+}$. Hence, $U_{+}$is unitary.

Equation (3.5) is an immediate consequence of the relation

$$
1-\theta_{\sigma r}(i, j)=\theta_{\sigma, \tau r}(i, j),
$$

so that the theorem is proved.

Proof of Theorem 2. We shall first prove (3.8) $)_{+}$. Define for $f \in \mathscr{H}$,

$$
\alpha_{f}(t)=\left\|\left(\exp (i \check{H} t) J \exp \left(-i H_{0} t\right)-U_{+}\right) f\right\| .
$$


Clearly, we need only show that $\alpha_{f}(t) \rightarrow 0$ for $t \rightarrow \infty$ for $f$ belonging to a total set. We shall prove this for $f \in \mathscr{D}$, defined by Eq. (3.10). Indeed, let $f \in \mathscr{D}$ and $\operatorname{supp} f \subset M_{\tau}$, then we can write, using the definition (3.7) of $\check{H}$,

$$
\alpha_{f}(t)=\left\|\left(U_{+}-J\right) \exp \left(-i H_{0} t\right) f\right\|=\|u(t, \cdot)\|,
$$

where

$$
\begin{aligned}
u(t, x) \equiv & (2 \pi)^{-N / 2} \sum_{\sigma} \chi_{\sigma}(x) \int_{M_{\tau}} d k\left(\exp \left[-i \phi \sum_{i<j} \theta_{\sigma \tau}(i, j) \epsilon\left(k_{i}-k_{j}\right)\right]-1\right) \\
& \cdot \exp \left(i k x-i \sum_{j=1}^{N} h\left(k_{j}\right) t\right) f(k) .
\end{aligned}
$$

Now the phase factor is stationary if and only if

$$
x=\left(h^{\prime}\left(k_{1}\right), \ldots, h^{\prime}\left(k_{N}\right)\right) t .
$$

Thus, taking $t>0$ we must have $x \in M_{\tau}$ if $k \in M_{\tau}$ for the phase factor to be stationary, since $h^{\prime}(k)$ is by assumption strictly increasing. Because supp $f$ stays away from the hyperplanes bounding $M_{\tau}$ it follows (cf. Ref. [1, Appendix XI.3.1]) that in the wedges $M_{\sigma}$ with $\sigma \neq \tau u(t, x)$ satisfies a bound

$$
|u(t, x)| \leqslant C_{N}\left(1+t^{2}\right)^{-N}\left(1+x^{2}\right)^{-N} .
$$

Hence, its $L^{2}$-norm over these wedges converges to zero for $t \rightarrow \infty$. But $u(t, x)$ vanishes for $x \in M_{\tau}$, since $\theta_{\tau \tau}(i, j)=0$. Therefore $\lim _{t \rightarrow \infty} \alpha_{f}(t)=0$, so that (3.8) follows.

To prove (3.8), we proceed similarly and get again (3.20)-(3.21), but with $U_{+} \rightarrow$ $U_{-},-\phi \rightarrow \phi$ and $\theta_{o \tau} \rightarrow \theta_{\sigma, \tau r}$. Taking $t<0$, we conclude using (3.22) that we must have $x \in M_{\tau r}$ for the phase to be stationary. But in this wedge the new $u(t, x)$ vanishes, so that (3.8). follows as before.

\section{Relativistic Dynamics Leading to the Ising and Federbush Model $S$-Matrices}

\section{A. The Continuum Ising Model}

The field theory associated to the Ising model in the scaling limit has been formulated in Ref. [8] in terms of formal field operators acting on a fermion Fock space, but the field operators describe a system of neutral bosons with mass $m>0$ and without bound states. Assuming one could formulate this field theory along the lines of axiomatic field theory [35-37], a description of the Haag-Ruelle theory in terms of wave operators [1] leads to an $S$-matrix on a boson Fock space, which can be taken to be $\mathscr{F}_{s} \equiv \oplus_{N=0}^{\infty} L_{s}^{2}\left(R^{N} ; d k_{1} \cdots d k_{N}\right)$. The $S$-operator obtained in Ref. [8] acts as multiplication by $(-)^{N(N-1) / 2}$ on the $N$-particle sector in $\mathscr{F}_{s}$. 
To construct a relativistic dynamics with this $S$-matrix we shall use the results of the preceding section: Define unitary operators $S, J, U_{ \pm}$and self-adjoint operators $H_{0}, \breve{H}$ on Fock spaces $\mathscr{F} \equiv \bigoplus_{N=0}^{\infty} L^{2}\left(R^{N} ; d k_{1} \cdots d k_{N}\right)$ and $\mathscr{F} \equiv \oplus_{N=0}^{\infty} L^{2}\left(R^{N} ; d x_{1} \cdots\right.$ $\left.d x_{N}\right)$ by letting them act on the $N$-particle sector $(N>0)$ like in Section 3 and by setting them equal to one resp. zero on the vacuum. The resulting classes of operators $H_{0}, \breve{H}$ will be again denoted $\mathscr{C}_{0}, \breve{\mathscr{C}}$. Note that $H_{0}$ equals the operator that is customarily denoted by $d \Gamma(h(k))$. Taking $\phi=\pi$ from now on we observe that our $S$ equals $(-)^{N(N-1) / 2}$ on the $N$-particle sector in $\mathscr{F}$. Moreover, $U_{ \pm}$and $S$ map the (anti-) symmetric wave functions onto themselves, so that all operators leave the fermion and boson Fock spaces contained in $\mathscr{F}$ resp. $\mathscr{F}$ invariant. (Note this is only true if $\phi=n \pi$, $n \in Z$.) We now define the following representation of the Poincare group on the oneparticle space $L^{2}(R ; d k)$ :

$$
\begin{aligned}
\left(U_{f}(a, \Lambda) g\right)(k)= & {\left[\left(E_{k} \cosh \alpha-k \sinh \alpha\right) / E_{k}\right]^{1 / 2} } \\
& \cdot \exp \left(i a_{0} E_{k}-i a_{1} k\right) g\left(k \cosh \alpha-E_{k} \sinh \alpha\right),
\end{aligned}
$$

where

$$
A \equiv\left(\begin{array}{ll}
\cosh \alpha & \sinh \alpha \\
\sinh \alpha & \cosh \alpha
\end{array}\right)
$$

and

$$
E_{k}=\left(k^{2}+m^{2}\right)^{1 / 2}
$$

The second quantization $\Gamma\left(U_{f}(a, \Lambda)\right)$ on $\mathscr{F}$ will be denoted by $U_{f}(a, \Lambda)$ as well. Clearly, $U_{f}(a, \Lambda)$ is a unitary positive energy representation. We can then define an interacting representation by setting

$$
\breve{I}_{i}(a, \Lambda) \equiv U_{ \pm} U_{f}(a, \Lambda) U_{ \pm}^{-1} .
$$

Since $U_{f}$ commutes with $S$ this definition does not depend on whether one takes $U_{+}$ or $U_{-}$. Also, in view of the above, $\breve{U}_{i}(a, \Lambda) \mathscr{F}_{s}=\check{\mathscr{H}}_{s}$. Denoting the free resp. interacting time translation generator by $H_{f}$ resp. $\check{H}_{i}$, Theorem 2 implies that

$$
\operatorname{s}_{t \rightarrow \pm \infty} \operatorname{limp}\left(i \breve{H}_{i} t\right) J \exp \left(-i H_{f} t\right)=U_{ \pm}
$$

so by virtue of (3.5) the operator $S$ is the $S$-operator associated to the pair $\check{H}_{i}, H_{f}$.

Summarizing, we have shown that there exist a representation $\breve{U}_{i}(a, \Lambda)$ of the Poincaré group on $\check{\mathscr{F}}_{s}$ and a corresponding free representation $U_{f}(a, \Lambda)$ on $\mathscr{F}_{s}$, such that the wave operators (4.5) exist and are unitary, and intertwine $U_{f}(a, \Lambda)$ and $\breve{U}_{i}(a, \Lambda)$. The resulting $S$-operator is the one of the Ising model field theory. Moreover, the operators $U_{ \pm}$and $S$ are the wave and scattering operators for any pair $\breve{H}, H_{0}$ in the classes $\check{\mathscr{C}}, \mathscr{C}_{0}$ defined above.

It is easy to see that the space translation generators $P_{i}$ and $P_{f}$ are equal, so that the interacting representation $U_{i}(a, \Lambda)$ is of the instant form in Dirac's terminology [86]. (Remember that $A \equiv J^{-1} \breve{A} J$.) It is also possible to construct an interacting dynamics 
of the point form, i.e., such that the boost generators are equal. To this end, let us regard the $k_{i}$ as particle rapidities, and correspondingly define a free representation $U_{f}^{\prime}(a, \Lambda)$ on $\mathscr{F}$ as the second quantization of the one-particle representation

$$
\left(U_{f}^{\prime}(a, \Lambda) g\right)(k)=\exp \left(i a_{0} m \cosh k-i a_{1} m \sinh k\right) g(k-\alpha),
$$

where $A$ and $\alpha$ are again connecled by (4.2).

Defining

$$
\check{U}_{i}^{\prime}(a, \Lambda)=U_{ \pm} U_{f}^{\prime}(a, \Lambda) U_{ \pm}^{-1}
$$

it is easy to see that the same situation obtains as in the case of $U_{f}$ and $\check{U}_{i}$, but in this case the boost generators $L_{i}^{\prime}$ and $L_{f}^{\prime}$ are equal.

In a previous paper [45] we studied a scheme for a relativistic point form dynamics. We would like to point out that on the two-particle sector the operator $H_{i}^{\prime}$ cannot be written in a form $H_{f}^{\prime}+P$ as considered in Subsection $2 \mathrm{~A}$ of that paper. Indeed, the intersection of the domains of $\check{H}_{f}^{\prime}$ and $\check{H}_{i}^{\prime}$ on $L^{2}\left(R^{2} ; d x_{1} d x_{2}\right)$ consists of the zero vector, since functions in the former domain are real-analytic, while functions in the latter domain are not, as is readily seen from the definition of $U_{ \pm}$. The same remark applies to $H_{f}^{\prime 1 / 2}$ and $H_{i}^{\prime 1 / 2}$, so that one cannot have $H_{i}^{\prime}=H_{f}^{\prime}+P$ in the sense of quadratic forms either. Note also that the same situation obtains on the $N$-particle sector with $N>2$.

\section{B. The Federbush Model}

The Federbush model describes a system of two species (I and II) of massive charged fermions without bound states. Hence, its asymptotic states may be described by wave functions satisfying

$$
\sum_{N=0}^{\infty} \sum_{s_{i}= \pm 1} \sum_{\delta_{i}= \pm 1} \int d k_{1} \cdots d k_{N}\left|\psi\left(k_{1}, s_{1}, \delta_{1} ; \ldots ; k_{N}, s_{N}, \delta_{N}\right)\right|^{2}<\infty
$$

and antisymmetric under permutations of the $\left(k_{i}, s_{i}, \delta_{i}\right)$. Here, $s=1(-1)$ denotes species I (II), while $\delta=1(-1)$ denotes positive (negative) charge. Let us first assume that $m_{\mathrm{I}}=m_{\mathrm{II}}=m$, and regard the $k_{i}$ as momenta. Then the Federbush $S$-matrix is the multiplication operator

$$
\begin{gathered}
(S \psi)\left(k_{1}, s_{1}, \delta_{1} ; \ldots ; k_{N}, s_{N}, \delta_{N}\right)=\exp \left[-\pi i \lambda \sum_{i<j} \epsilon\left(k_{i}-k_{j}\right)\left(s_{i}-s_{j}\right) \delta_{i} \delta_{j}\right] \\
\cdot \psi\left(k_{1}, s_{1}, \delta_{1} ; \ldots ; k_{N}, s_{N}, \delta_{N}\right),
\end{gathered}
$$

where $\lambda$ is the coupling constant multiplying the interaction Lagrangean [10].

Clearly, we can also define $S$ by (4.9) on the Fock space $\mathscr{F}$ of wave functions satisfying (4.8), but not necessarily antisymmetric in the arguments $\left(k_{i}, s_{i}, \delta_{i}\right)$. We can then proceed as in the Ising case to define operators $J, U_{ \pm}, H_{0}, \check{H}$ on the Fock spaces $\mathscr{F}$ and $\mathscr{F}$, using the definitions (3.9), (3.3)-(3.4), (3.6), (3.7), but replacing $\epsilon\left(k_{i}-k_{j}\right)$ 
by $\epsilon\left(k_{i}-k_{j}\right)\left(s_{i}-s_{j}\right) \delta_{i} \delta_{j}, \phi$ by $-\pi \lambda$ and adding the internal quantum numbers $s_{i}, \delta_{i}$ in the arguments of the wave functions. Thus, e.g.,

$$
\begin{gathered}
\left(U_{-} \psi\right)\left(x_{1}, s_{1}, \delta_{1} ; \ldots ; x_{N}, s_{N}, \delta_{N}\right) \\
=(2 \pi)^{-N / 2} \sum_{\sigma, \tau} \chi_{\sigma}(x) \cdot \int d k \exp \left[-\pi i \lambda \sum_{i<j} \theta_{\sigma, \tau r}(i, j) \epsilon\left(k_{i}-k_{j}\right)\left(s_{i}-s_{j}\right) \delta_{i} \delta_{j}\right] \\
\cdot \exp (i k x) \chi_{\tau}(k) \psi\left(k_{1}, s_{1}, \delta_{1} ; \ldots ; k_{N}, s_{N}, \delta_{N}\right) .
\end{gathered}
$$

It is straightforward to modify the proofs of Theorems 1 and 2 so as to obtain the analogous results for the operators on $\mathscr{\mathscr { F }}$ we have just defined. It is also readily seen that the $U_{ \pm}$leave the (anti-) symmetric wave functions invariant, so that one may

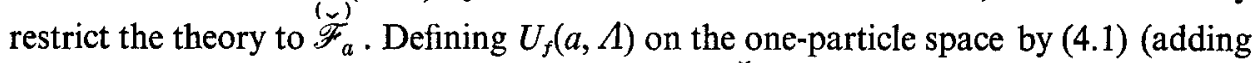
$s, \delta$ to the arguments) and subsequently defining $\breve{U}_{i}(a, \Lambda)$ by $(4.4)$, one is in the same situation as in the Ising case and the comments made there are applicable here as well. In particular one can also regard the $k_{i}$ as particle rapidities and define $U_{f}^{\prime}(a, \Lambda)$ and $U_{i}^{\prime}(a, \Lambda)$ as in the Ising case, which results in a point form dynamics.

The unequal mass case is a little more complicated. First, if one regards the $k_{i}$ as momenta, $S$ is no longer given by (4.9), since $k_{\mathrm{I}}>k_{\mathrm{II}}$ no longer implies particle $\mathrm{I}$ is to the right of particle II in the distant future if $m_{\mathrm{I}} \neq m_{\mathrm{II}}$. (Another way of seeing this is to note that $S$ would not commute with Lorentz boosts if it were given by (4.9) for $m_{\mathrm{I}} \neq m_{\mathrm{II}}$.) However, this difficulty does not occur if one considers the $k_{i}$ as rapidities, which we shall therefore do from now on. Correspondingly, we define $U_{f}(a, \Lambda)$ as the second quantization of the operator

$$
\left(U_{f}(a, \Lambda) g\right)(k, s, \delta)=\exp \left(i a_{0} m(s) \cosh k-i a_{1} m(s) \sinh k\right) g(k-\alpha, s, \delta)
$$

on the one-body space, where

$$
m(1)=m_{\mathrm{I}}, \quad m(-1)=m_{\mathrm{II}} .
$$

Now one might of course again define $J, U_{ \pm}$and $\check{U}_{i}(a, \Lambda)$ as in the equal mass case, but then it no longer follows as before that $U_{ \pm}$are the wave operators associated to $\breve{H}_{i}, H_{f}$. Indeed, the stationary phase argument in the proof of Theorem 2 hinges on having just one function $h(k)$, while our $H_{f}$ acts as

$$
\begin{aligned}
\left(H_{f} \psi\right) & \left(k_{1}, s_{1}, \delta_{1} ; \ldots ; k_{N}, s_{N}, \delta_{N}\right) \\
\quad= & \sum_{j=1}^{N} m\left(s_{j}\right) \cosh k_{j} \psi\left(k_{1}, s_{1}, \delta_{1} ; \ldots ; k_{N}, s_{N}, \delta_{N}\right),
\end{aligned}
$$

giving rise to two different functions $m_{\mathrm{I}} \cosh k, m_{\mathrm{II}} \cosh k$. However, let us redefine $J$ and $U_{ \pm}$by making the substitution

$$
\exp (i k x) \rightarrow \prod_{j=1}^{N} m\left(s_{j}\right)^{1 / 2} \exp \left(i m\left(s_{j}\right) k_{j} x_{j}\right)
$$


on the right-hand side of Eq. (4.10), and in the equations for $J$ and $U_{+}$. The resulting $J$ is again unitary, since it is the product of a unitary scale transformation and Fourier transformation. A scrutiny of the proof of Theorem 1 shows that the new $U_{ \pm}$are unitary too, and satisfy (3.5). Now define $\mathscr{C}_{0}$ as the class of multiplication operators $H_{0}=d \Gamma\left(\oplus_{s= \pm_{1}} m(s) h(k)\right)$, and the corresponding $\check{H} \in \check{\mathscr{C}}$ by (3.7). Similarly, define $\breve{U}_{i}(a, \Lambda)$ by (4.4).jThen aninspection of the proof of Theorem 2 shows that the analogs of the statements in that theorem also hold for the operators just defined. Again, one easily verifies that $U_{ \pm}$map the fermion and boson Fock spaces onto themselves, so that one can restrict the theory to $\check{\mathscr{F}}_{a}$. Thus, as before, we obtain a relativistic dynamics on $\mathscr{F}_{a}$ whose wave operators intertwine the interacting representation $\breve{U}_{i}(a, \Lambda)$ on $\breve{F}_{a}$ with the free representation $U_{f}(a, \Lambda)$ on $\mathscr{F}_{a}$ corresponding to the Federbush model, and whose $S$-matrix coincides with the Federbush $S$-matrix (4.9); this dynamics is singled out by invariance considerations from a large class of dynamics for which $U_{ \pm}$ and $S$ are the wave and scattering operators.

\section{ACKNOWLEDGMENTS}

The author is indebted to A. S. Wightman for drawing his attention to integrable field theories and for enlightening and stimulating discussions. He has also benefited from conversations with $\mathbf{M}$. Aizenmann, N. Andrei, D. Campbell, W. Garber, D. Gross, E. Lieb, J. Lowenstein, R. Newton, S. Oxford, R. Pisarski, H. Segur, E. Seiler, B. Simon, and L. Thomas. Part of the work in Ref. [47] mentioned in Section 2 was done at the Aspen Center for Physics during the summer of 1978. We would like to thank the Center for its hospitality.

\section{REFERENCES}

1. M. Reed And B. Simon, "Methods of Modern Mathematical Physics," Vol. III, "Scattering Theory," Academic Press, New York, 1979.

2. M. Reed and B. Simon, "Methods of Modern Mathematical Physics," Vol. I, "Functional Analysis," Academic Press, New York, 1972.

3. M. Reed AND B. Simon, "Methods of Modern Mathematical Physics," Vol. IV, "Analysis of Operators," Academic Press, New York, 1978.

4. R. Abraham AND J. E. MARSDEN, "Foundations of Mechanics," Benjamin/Cummings, Reading, Mass., 1978.

5. J. Moser, "Various Aspects of Integrable Hamiltonian Systems," Lectures at the CIME Summer Session in Dynamical Systems, Bressanone, Italy, 1978.

6. J. Moser, Advances in Math. 16 (1975), 197-220.

7. D. Kazhdan, B. Kostant, and S. Sternderg, Commun. Pure Appl. Math. 31 (1978), 481-507.

8. M. Sato, T. Miwa, AND M. Jimbo, Field theory of the 2-dimensional Ising model in the scaling limit, RIMS preprint 207, 1976.

9. P. Federbush, Phys. Rev. 121 (1961), 1247-1249.

10. B. Schroer, T. T. Truong, ANd P. Weisz, Ann. Phys. (N.Y.) 102 (1976), 156-169.

11. N. Andrei And J. H. Lowenstein, Phys. Rev. Lett. 43 (1979), 1698-1701; Phys. Lett. B 90 (1980), 106-110.

12. E. H. Lieb And W. Liniger, Phys. Rev. 130 (1963), 1605-1616.

13. M. Gaudin, J. Math. Phys. 12 (1971), 1674-1676. 
14. M. Gaudin, J. Math. Phys. 12 (1971), 1677-1680.

15. S. OXFord, "The Hamiltonian of the Quantized Nonlinear Schrödinger Equation," Ph.D. thesis, Los Angeles, 1979.

16. F. A. Berezin ANd V. N. Sushko, Sov. Phys. JETP 21 (1965), 865-873.

17. H. Bergknoff and H. B. Thacker, Phys. Rev. Lett. 42 (1979), 135-138.

18. H. Bergknoff and H. B. ThaCKer, Phys. Rev. D 19 (1979), 3666-3681.

19. H. BeTHe, Z. Phys. 71 (1931), 205-226.

20. L. ThOMAS, J. Math. Anal. Appl. 59 (1977), 392-414.

21. D. Babbitt AND L. Thomas, Proc. Nat. Acad. Sci. USA 74 (1977), 816-817.

22. D. Babbitt and L. Thomas, Commun. Math. Phys. 54 (1977), 255-278.

23. D. Babbitt and L. Thomas, J. Math. Phys. 19 (1978), 1699-1704.

24. D. Babbitt and L. Thomas, J. Math. Anal. 72 (1979), 305-328.

25. J. B. MCGuIRE, J. Math. Phys. 5 (1964), 622-636.

26. E. Brézin, J. Zinn-Justin, C. R. Acad. Sci. Paris 263 (1966), 670-673.

27. C. N. YANG, Phys. Rev. 168 (1968), 1920-1923.

28. A. B. Zamolodchikov and A. B. Zamolodchikov, Nucl. Phys. B 133 (1978), 525-535.

29. A. B. Zamolodchikov, Commun. Math. Phys. 55 (1977), 183-186.

30. A. B. ZaMOLODCHIKov, preprint ITEP-12, 1977.

31. M. Karowski and H. J. Thun, Nucl. Phys, B 130 (1977), 295-308.

32. P. H. WeISZ, Phys. Lett. B 67 (1977), 179-182.

33. M. Karowski, Phys. Reps. 49 (1979), 229-237.

34. L. HÖRMANDER, Math. Z. 146 (1976), 69-91.

35. R. F. Streater AND A. S. Wightman, "PCT, Spin and Statistics, and All That," Benjamin, New York, 1964.

36. R. Jost, "The General Theory of Quantized Fields," Amer. Math. Soc., Providence, R.I., 1965.

37. B. Simon, "The $P(\phi)_{2}$ Euclidean (Quantum) Field Theory," Princeton Univ. Press, Princeton, N.J., 1974.

38. J. FröHLICH, Commun. Math. Phys. 47 (1976), 269-310.

39. J. FröHLICH, Quantum sine-Gordon equation and quantum solitons in two space-time dimensions, in "Renormalization Theory" (G. Velo and A. S. Wightman, Eds.), pp. 317-415, Reidel, Dordrecht, 1976.

40. B. M. McCox, C. A. Tracy, ANd T. T. WU, Phys. Rev. Lett. 38 (1977), 793-796.

41. M. Sato, T. Miwa, AND M. Jimbo, Holonomic quantum fields, IV, RIMS preprint-263, 1978.

42. A. S. WIGHTMAN, Introduction to some aspects of the relativistic dynamics of quantized fields, in "High Energy Electromagnetic Interactions and Field Theory" (M. Lévy, Ed.), pp. 171-289, Gordon \& Breach, New York, 1967.

43. J. L. Challifour and A. S. Wightman, unpublished manuscript.

44. J. L. Challifour, J. Math. Phys. 9 (1968), 1137-1145.

45. S. N. M. RuiJsenaArs, Ann. Phys. (N.Y.) 126 (1980), 399-449.

46. "Constructive Quantum Field Theory" (G. Velo and A. S. Wightman, Eds.) Lecture Notes in Physics No. 25, Springer-Verlag, Berlin, 1973.

47. S. N. M. RuIJSENAARs, unpublished.

48. R. F. Dashen, B. Hasslacher, and A. Neveu, Phys. Rev. D 11 (1975), 3424-3450.

49. S. Coleman, Phys. Rev. D 11 (1975), 2088-2097.

50. B. Berg, M. Karowski, P. Weisz, And V. KuraK, Nucl. Phys. B 134 (1978), 125-132.

5l. V. E. Zakharov and S. V. ManaKov, Theor. Math. Phys. 19 (1974), 551-559.

52. H. B. THACKer, Phys. Rev. D 17 (1978), 1031-1040.

53. J. H. Lowenstein AND E. R. SPeer, Commun. Math. Phys. 63 (1978), 97-112.

54. J. H. Lowenstern and E. R. Speer, Nucl. Phys. B 158 (1979), 397-409.

55. L. A. Takhtadzhyan and L. D. FaddeEv, Theor. Math. Phys. 21 (1974), 1046-1057.

56. E. A. KuZnetsov and A. V. Mikhailov, Theor. Math. Phys. 30 (1977), 193-200.

57. D. W. Mclaughlin, J. Math. Phys. 16 (1975), 96-99. 
58. H. F. FlaschKa AND A. C. Newell, Integrable systems of nonlinear evolution equations, in "Dynamical Systems, Theory and Applications" (J. Moser, Ed.), pp. 355-440, Lecture Notes in Physics No. 38, Springer-Verlag, Berlin, 1975.

59. V. E. Zakharov and L. D. Faddeev, Func. Anal. Appl. 5 (1971), 280-287.

60. A. Neveu and N. Papanicolaou, Commun. Math. Phys. 58 (1978), 31-64.

61. K. Pohlmeyer, Commun. Math. Phys. 46 (1976), 207-221.

62. C. S. Gardner, J. M. Greene, M. D. Kruskal, And R. M. Miura, Phys. Rev. Lett. 19 (1967), 1095-1097.

63. A. C. Scott, F. Y. F. Chu, and D. W. McLaughlin, Proc. IEEE 61 (1973), 1443-1483.

64. M. J. Ablowitz, D. J. Kaup, A. C. Newell, and H. Segur, Stud. Appl. Math. 53 (1974), 249-315.

65. P. P. Kulish, S. V. Manakov, and L. D. Faddeev, Theor. Math. Phys. 28 (1976), 615-620.

66. C. S. Gardner, J. M. Greene, M. D. Kruskal, and R. M. Miura, Commun. Pure Appl. Math. 27 (1974), 97-133.

67. H. B. Thacker and D. Wilkinson, Phys. Rev. D 19 (1979), 3660-3665.

68. J. Honerkamp, P. Weber, AND A. Wiesler, Nucl. Phys. B 152 (1979), 266-272.

69. R. Hirota, J. Phys. Soc. Japan 33 (1972), 1459-1463.

70. BaXter, R. J., Ann. Phys. (N.Y.) 70 (1972), 323-337.

71. J. D. Johnson, S. Krinsky, AND B. M. McCoy, Phys. Rev. A 8 (1973), 2526-2547.

72. A. Luther, Phys. Rev. B 14 (1976), 2153-2159.

73. M. LüsCher, Nucl. Phys. B 117 (1976), 475-492.

74. S. N. M. RuIJSENAARs, The continuum limit of the infinite isotropic Heisenberg chain in its ground state representation, J. Func. Anal. 39 (1980), 15-84.

75. J. HoNerkamp AND P. WeBer, Calculation of the $S$-matrix from the Hamiltonian of the massive Thirring model, Freiburg preprint, 1979.

76. D. IAgolnitzer, Phys. Rev. D 18 (1978), 1275-1285.

77. D. IAgolnitzer, Phys. Lett. B 76 (1978), 207-209.

78. M. J. Ablowitz, A. Ramani, And H. Segur, Lett. Nuovo Cim. 23 (1978), 333-338.

79. M. J. Ablowitz, A. Ramani, And H. Segur, J. Math. Phys. 21 (1980), 715-721, 1006-1015.

80. E. H. Wichmann ANd J. H. Crichton, Phys. Rev. 132 (1963), 2788-2799.

81. W. Hunziker, J. Math. Phys. 6 (1965), 6-10.

82. K. Hepp, Helv. Phys. Acta 37 (1964), 659-662.

83. S. Coleman and J. Mandula, Phys. Rev. 159 (1967), 1251-1256.

84. J. T. LopuszansKi, J. Math. Phys. 12 (1971), 2401-2412.

85. W. D. Garber and H. Reeh, Commun. Math. Phys. 70 (1979), 169-180.

86. P. A. M. DiraC, Rev. Mod. Phys. 21 (1949), 392-399. 